\title{
LAS ESTRATEGIAS EN AGRUPACIONES APÍCOLAS DE LA PROVINCIA DE SANTA FE Y ESTE DE CÓRDOBA (ARGENTINA) $^{\mathrm{a}}$
}

\author{
Travadelo, m. ${ }^{1}$; Suero, M. ${ }^{2} ;$ Maina, M. ${ }^{3}$; Brizi, M. C. ${ }^{4}$; Rossler, N. ${ }^{5}$; \\ Ulmer, J. ${ }^{6} \&$ CAPORgno, J. ${ }^{7}$
}

\begin{abstract}
RESUMEN
El objetivo de esta investigación es conocer el comportamiento estratégico de las diferentes agrupaciones apícolas que operan en el ámbito de la provincia de Santa Fe y Este de Córdoba. La metodología utilizada fue la de estudios de casos múltiples, seleccionados por informantes calificados.

El análisis de las entrevistas realizadas permitió reunir las organizaciones en 2 grupos de acuerdo al mercado de destino principal donde los apicultores entregan su producción: venta directa a importador o mediante un exportador/acopiador. Las opciones estratégicas seguidas por cada organización se analizan bajo el marco analítico propuesto por Ansoff (1988).

En el proceso de pensar las estrategias se promueve una mayor integración de los socios, activa la evolución y adaptación y favorece el logro de los objetivos organizacionales; fomenta la confianza y lealtad, bases cognitivas en la construcción del capital social de cada organización. Trabajar en el seno de las mismas es esencial para su supervivencia.
\end{abstract}

Palabras claves: apicultura, agrupaciones, economía social, planificación estratégica, estrategia corporativa.

\footnotetext{
a.- En el Marco del Proyecto CAID 2009 No 12/C119 - Universidad Nacional del Litoral. Directora: Mariana Travadelo.

1.- Profesora Titular de Mercadeo Agroalimentario. Facultad de Ciencias Agrarias. Universidad Na-cional del Litoral. Telefax 54-3496-420639. E-mail: mtravade@fca.unl.edu.ar. Kreder 2805 (3080) Esperanza, Santa Fe. Argentina.

2.- Jefe de Trabajos Prácticos de Administración de Organizaciones. FCA/UNL)

3.- Ayudante de Cátedra de Mercadeo Agroalimentario. FCA (UNL).

4.- Ayudante de Cátedra de Administración de Organizaciones. FCA (UNL)

5.-Adscripto Graduado en docencia en la asignatura Administración de Organizaciones. FCA (UNL).

6.- Instituto Nacional de Tecnología Agropecuaria. EEA Rafaela. AER Ceres (Santa Fe- Argentina).

Manuscrito recibido el 19 de marzo de 2013 y aceptado para su publicación el 24 de junio de 2013.
} 


\section{SUMMARY}

\section{Strategies in beekeeping groups of Santa Fe Province and East of Córdoba (Argentina).}

The aim of this research is to understand the strategic behavior of different beekeeping groups operating in provinces of Santa Fe and East of Cordoba. The methodology used was a multiplecase study selected by informants.

The analysis of the interviews brought together the organizations into 2 groups according to the main target market where beekeepers deliver their production: direct sales to importers, or through middlemen-exporters. The strategic options pursued by each organization are analyzed under the analytical framework proposed by Ansoff (1988).

In the process of thinking the strategies is promoted greater integration of partners, is enabled the evolution and adaptation and is fostered the achievement of organizational objectives, builds trust and loyalty, building knowledge bases in capital organization. Work within them is essential to its survival. Key words: beekeeping, groups, social economy, strategic planning, corporative strategy.

\section{INTRODUCCIÓN}

Las agrupaciones en la actividad apícola juegan un papel trascendente para insertar la producción primaria en la cadena de valor del producto, dado que la mayoría de los apicultores producen a pequeña escala, a tiempo parcial y exhiben una gran dispersión.

Los beneficios de la acción colectiva realizada a través de las agrupaciones han sido ampliamente justificados con suficiente evidencia empírica (Uphoff \& Wijayaratna, 2000; Reardon et al., 2005; Reardon et al., 2008; Barham \& Chitemi, 2009; Hellin et al., 2009). La superación de altas barreras de entrada y la disminución de los costos de transacción para acceder a insumos y productos, son algunas de las ventajas que las organizaciones de productores obtienen y facilitan el acceso a mercados, posibilitando la inserción eficaz en las cadenas de valor (Pingali et al., 2005; Poulton et al., 2005; Markelova, 2008).

Aunque las organizaciones de productores no proveen claros beneficios en el acceso a los mercados de commodities sin diferenciación (Hellin et al., 2009), sí lo mejoran particularmente en productos agrícolas con mayor valor agregado por actividades de procesamiento o a través de comercialización conjunta. También pueden contribuir al bienestar de sus miembros ofreciendo otros servicios (Travadelo et al., 2012), facilitando el acceso a innovaciones e información y buscando oportunidades de generar valor agregado al producto o de diferenciarlo para poder acceder a nuevos mercados. Hay evidencia empírica que la acción colectiva puede ayudar a los productores a reducir las barreras de entrada, aumentando su poder de negociación frente a compradores e intermediarios, mejorando la coordinación entre la oferta y la demanda (Thorp et al. 2005; Markelova et al., 2009).

La planificación estratégica es uno de los pasos de la dirección estratégica que orienta y focaliza el devenir ordenado de una organización. Permite identificar el/los caminos que son necesarios recorrer para poder alcanzar los objetivos organizacionales (Robbins \& Coulter, 2005). Novkovic \& Power (2005) y Martín López \& Ruíz Gue- 